\title{
PERCEPCIÓN DE LOS ESTUDIANTES DE EDUCACIÓN BÁSICA MUNICIPALIZADOS SOBRE LA ENSEÑANZA DE LA MATEMÁTICA
}

\author{
Perception of municipalized elementary school students \\ about the teaching of Mathematics
}

https://doi.org/10.22235/pe.v10i2.1425

\author{
ALEJANDRO SEPÚLVEDA OBREQUE*1 \\ CARLOS OYARZÚN BURGOS*2 \\ DANILO DÍAZ-LEVICOY**3 \\ MARGARITA OPAZO SALVATIERRA***4
}

*Universidad de Los Lagos. Chile.

Correspondencia: asepulve@ulagos.cl, coyarzun@ulagos.cl

Recibido: 01/02/17

**Universidad de Granada. España.

Correspondencia: dddiaz01@hotmail.com

Revisado: 16/03/17

${ }^{* * *}$ Universidad de Playa Ancha. Chile.

Aceptado: $28 / 04 / 17$

Correspondencia:mopazos@gmail.com

1 Profesor de Ciencias Naturales y Biología (Universidad Católica de Chile). Magíster en Administración Educacional (Universidad de Antofagasta). Doctorado en Educación (Universidad Humanismo Cristiano-PIIE). Sus principales líneas de investigación son la Didáctica de las Ciencias y evaluación auténtica del aprendizaje. Académico en el Departamento de Educación de la Universidad de Los Lagos (Chile).

2 Educador Diferencial (Universidad Austral de Chile). Magíster en Educación Especial (Universidad Católica de Chile). Sus principales líneas de investigación son la Didáctica de la Matemática y las dificultades de aprendizaje. Académico en el Departamento de Educación de la Universidad de Los Lagos (Chile).

3 Profesor de Educación Media en Matemática y Computación (Universidad de Los Lagos). Máster Universitario en Didáctica de la Matemática (Universidad de Granada). Sus principales líneas de investigación son la Didáctica de la Matemática y Didáctica de la Estadística. Doctorando en Ciencias de la Educación por la Universidad de Granada (España).

4 Profesora de Educación Básica (Universidad de Playa Ancha). Magister en Orientación Educacional (Universidad de Playa Ancha). Sus principales líneas de investigación son la gestión educacional, competencias transversales y la evaluación del aprendizaje. Académica en la Facultad de Educación de la Universidad de Playa Ancha (Chile). 
Resumen: Este artículo tiene como propósito conocer la opinión de estudiantes sobre la enseñanza de la matemática. Para ello, se aplicó un cuestionario a 768 estudiantes de séptimo y octavo año de Educación Básica de dos ciudades en la región de Los Lagos (Chile). Los resultados muestran que los estudiantes perciben a sus profesores como exigentes, comprensivos y dispuestos al diálogo, y ponderan de manera positiva las capacidades de estos para explicar una misma materia de manera distinta y presentar los contenidos de forma atrayente. Además, observan que sus profesores se comprometen con el logro de los aprendizajes; advierten sus esfuerzos genuinos y sistemáticos para que los alumnos aprendan y valoran las interacciones profesor-alumno, pues tienen como objetivo la comprensión de lo enseñado.

Palabras clave: enseñanza, matemática, estudiantes, profesores, educación básica.

Abstract: This article focuses in revealing student's opinions on Mathematics teaching. In order to gather the information, a questionnaire was given to 768 students of seventh and eighth of basic education from Los Lagos (Chile). The results show that students who perceive their teachers as demanding, comprehensive and willing to dialogue, qualify in a positive way their capacity to explain the same subject on a different and interesting way. They see their teachers committed with the learning goals of different topics; they notice their genuine and systematic efforts for their students to learn; they assess positively the student-teacher interaction since their purpose is the comprehension.

Keywords: teaching, Mathematics, students, teachers, basic education.

\section{INTRODUCCIÓN}

Investigar sobre el desempeño del profesor en su práctica puede ayudar a interpretar mejor el trabajo del estudiante, encontrar maneras de ayudar al docente a desarrollar competencias necesarias para ejercer mejor su profesión y, también, facilitar la elaboración de planes de formación más acordes con las características de los docentes (Blanco, 1997). De acuerdo con lo anterior, asiste el interés de estudiar la enseñanza de la matemática en la Educación Básica desde la perspectiva de los estudiantes, ya que ha sido una materia complicada para muchos de ellos.

De acuerdo con Prado, Navarro, Berguido y De la Cruz (2013), para un número importante de estudiantes la enseñanza de la matemática es aburrida, agobiante $y$, en general, motivo de frustración y fracaso. Muchos no tienen interés en estudiarla porque se enseña de forma monótona, sin actividades lúdicas; lo que genera apatía y redunda en bajos niveles de comprensión y dificultades para aprender. En consecuencia, existe una gama de razones que determina el desinterés por esta asignatura (Prado et al., 2013). 
Según el Programa Internacional de Evaluación de los Alumnos (PISA) de la Organización para el Crecimiento y el Desarrollo Económico (OECD; 2004), a los estudiantes les interesa más la lectura que la matemática. Este informe da cuenta de que uno de cada tres estudiantes de los países de la OECD coincide en que se ponen muy nerviosos, inquietos e incluso se bloquean cuando tienen que resolver problemas de matemática.

Pues bien, el presente estudio se centra específicamente en la enseñanza de esta asignatura, ya que es fundamental para el desarrollo intelectual de los estudiantes; les ayuda a ser lógicos, a razonar de forma ordenada y a tener una mente preparada para el pensamiento, la crítica y la abstracción (Organización de Estados Iberoamericanos, 2015). El aprendizaje de la matemática promueve la formación de valores, actitudes y conductas en los estudiantes, ya que es un referente para orientar la vida de los alumnos, como lo son el razonamiento y abstracción, la comprensión, la búsqueda de información, el empleo de simbología (Reimers, 2006).

A pesar de su importancia, necesidad de conocerla y comprenderla, es ampliamente sabido que los aprendizajes escolares en el área son insuficientes y que los estudiantes, al menos una buena parte de ellos, no consiguen construir conceptos matemáticos complejos y utilizarlos en situaciones nuevas (Badano y Dodera, 1999; Bolea, Bosch y Gascón, 2001; Corica, 2007; Gascón, Bosch y Bolea, 2001; Míguez, 2004; Otero, Fanaro y Elichiribehety, 2001).

Se observa una tendencia descendente en el agrado e interés por la matemática en los estudiantes a medida que avanzan en los niveles educativos (Hidalgo, Maroto y Palacios, 2004). Esto se suma con que a los estudiantes, en particular los del primer ciclo de Educación Básica, les agrada la matemática pero, a la primera dificultad, desisten y recurren a pedirle ayuda al profesor, quien en lugar de reorientar la pregunta les entrega la respuesta. Este hecho, según Corica y Otero (2007), demuestra que los estudiantes tienen baja resistencia a la frustración y que la forma de proceder del docente daña la actividad de aprendizaje.

Corica (2007) menciona que existen características del profesor de matemática que son valoradas por parte de sus estudiantes, y se refiere en especial a las capacidades didácticas para comunicar y transferir el conocimiento, junto con aspectos actitudinales y relacionales hacia los alumnos.

En función de estas premisas, en este estudio se evalúa cómo enseña el profesor desde la perspectiva de los estudiantes, ya que son una significativa fuente de información en la valoración del desempeño docente (Tejedor, 2003). Los datos aportados por ellos permiten observar características importantes de lo que sucede en clase, durante el proceso de instrucción, así como sobre la medida en la que se logran los objetivos establecidos por la institución educativa (Rojas y Cortés, 2002).

Se espera que los datos recabados sirvan de insumo para apoyar la reflexión y la toma de decisiones sobre la enseñanza de la matemática en la Educación Básica. 


\section{DISEÑO TEÓRICO DEL ESTUDIO}

\section{Planteamiento y formulación de la pregunta problema}

No hay duda de que la matemática está presente en la vida cotidiana de todas las personas; su uso se encuentra en muchos ámbitos del quehacer humano, desde las actividades diarias hasta la investigación científica. Se caracteriza por ser una de las asignaturas a la que se destina mayor atención en las unidades educativas, se imparte en todos los niveles del sistema educacional (Organización de Estados Iberoamericanos, 2015). Sin embargo, los resultados y niveles de logro que obtienen los estudiantes, en diferentes mediciones, son bajos (ej., SIMCE, PISA, TIMSS, PSU). Lo comentado lleva a preguntar: ¿cuál es la percepción que tienen los estudiantes sobre la enseñanza de la matemática?

Este artículo da cuenta de resultados que forman parte de un proyecto de mayor envergadura, que considera otros ámbitos: la evaluación del aprendizaje, las características del profesor de matemática en el aula, las razones para no aprender matemática en clase (Sepúlveda et al., 2016), la percepción de la asignatura en el aula, el compromiso del estudiante con el aprendizaje de la matemática en aula, el proceso evaluativo y los instrumentos utilizados en la enseñanza-aprendizaje de la materia (Sepúlveda, Payahuala, Lemarie y Opazo, 2017). El objetivo de este estudio, en concreto, es determinar la percepción sobre la enseñanza de matemática que tienen los estudiantes de séptimo y octavo año básico en escuelas municipalizadas de las comunas de Osorno y Frutillar (Los Lagos, Chile).

\section{MÉTODO}

Esta investigación se clasifica como no experimental, tipo encuesta (Labarca, 2001), de carácter descriptivo, de corte transversal y de procedimiento metodológico cuantitativo (Hernández, Fernández y Baptista, 2010).

\section{Participantes}

La muestra de estudiantes a los que se les aplicó el instrumento fue de 768 alumnos de séptimo y octavo año de once escuelas municipales de las comunas de Frutillar y Osorno. Dichos establecimientos fueron elegidos porque otorgaron la autorización correspondiente para aplicar el instrumento. Su dependencia es municipal, por lo que reciben subvención del Estado. En su mayoría, pertenecen a contextos educacionales, culturales y económicos medio bajos. Respecto de los participantes, el $49 \%$ son estudiantes de séptimo y el $51 \%$ de octavo año básico; un $48,3 \%$ son mujeres y un $51,7 \%$ son hombres. 
Se optó por estos niveles educativos por considerar que los estudiantes cuentan con experiencia suficiente para dar un juicio sobre su realidad escolar y porque son los cursos terminales del nivel en educativo en cuestión. Además, han tenido un número importante de profesores en sus aulas.

El tipo de muestra fue no probabilística de carácter accidental, es decir, se administró el instrumento a los estudiantes que se encontraban en el momento de la aplicación en las salas de clases.

\section{Técnicas e instrumentos de recolección de datos}

Dada la naturaleza del estudio y los objetivos de este, se definió la aplicación de una encuesta para recoger la información. Se diseñó, en primera instancia, un listado de preguntas que darían indicadores sobre la percepción de la enseñanza de la matemática que tienen los estudiantes.

El cuestionario quedó estructurado por veintiocho (28) preguntas de alternativas, con opción múltiple de entre tres y seis respuestas, como también se podía optar por más de una alternativa en algunas de ellas. Los ámbitos temáticos del instrumento fueron: (1) características del profesor de matemática en el aula; (2) proceso de enseñanza-aprendizaje de la matemática en aula; (3) razones para no aprender matemática en clase; (4) percepción de la matemática en el aula; (5) compromiso del estudiante con el aprendizaje de la matemática en aula; (6) proceso evaluativo e instrumentos utilizados en la enseñanza-aprendizaje de la matemática.

Cabe señalar que este artículo forma parte de un estudio de mayor envergadura y que corresponde a una parte de este. En consecuencia, las áreas temáticas analizadas fueron: características del profesor de matemática, percepción de los estudiantes y proceso enseñanza-aprendizaje de la matemática en aula; que responden específicamente a los objetivos del presente estudio.

\section{Validación del instrumento}

La validación interna del cuestionario fue a través de criterio de experto (3). La revisión del instrumento por parte de los expertos se refirió a aspectos de forma tales como redacción y puntuación, que fueron aceptadas e incorporadas. El instrumento mejorado fue aplicado a una muestra piloto de 35 estudiantes pertenecientes al mismo nivel educativo de la población estudiada. En general, los estudiantes que conformaban la muestra piloto no presentaron dificultades para dar respuesta a las distintas preguntas que el instrumento contenía. 


\section{Análisis de fiabilidad del cuestionario}

Para conocer la fiabilidad del cuestionario, se realizó el cálculo del coeficiente de fiabilidad (Alpha de Cronbach) y de los índices de homogeneidad de los ítems, con lo que se obtuvo la medida de su consistencia interna. El coeficiente de fiabilidad obtenido fue alto $(0,803$ de un máximo de 1$)$.

\section{Trabajo de campo}

Se inició con el consentimiento y la explicación de los objetivos del estudio a los directivos de las unidades educativas y el profesor jefe de los cursos participantes de la investigación.

El cuestionario fue aplicado por los investigadores y el profesor encargados de los cursos correspondientes, en las respectivas salas de clases, en las horas libres, entre los meses de abril y agosto de 2015. El tiempo de duración de la aplicación del instrumento fue de 25 minutos aproximadamente.

\section{Análisis y presentación de los datos}

Una vez recogidos, sistematizados y depurados los datos en las tablas de contingencia diseñadas, se procede a obtener la frecuencia y porcentaje de cada una de las alternativas de las preguntas del cuestionario. Luego se agrupan las preguntas y sus correspondientes resultados de acuerdo con tres categorías: características del profesor de matemática, percepción de los estudiantes y proceso enseñanzaaprendizaje de la matemática en aula.

\section{PRESENTACIÓN DE RESULTADOS}

\section{Características del profesor de matemática: percepción de los estudiantes}

Los estudiantes son una importante fuente de información, ya que permiten observar características de lo que ocurre en la sala de clase. En este caso, interesa conocer su opinión respecto de cómo perciben a su profesor con algunas de las siguientes características: paciente, exigente, aburrido, divertido, dialogante, comprensivo. Se observa (Figura 1) que las opiniones son positivas, como persona y como profesional. Destacan que es una persona que está disponible al diálogo $(55,8 \%)$ y, en relación con esta característica, lo definen como comprensivo (48,6\%), cualidades importantes en la enseñanza. Lo perciben también como una persona paciente $(40,8 \%)$, característica, se estima, muy importante para enseñar matemática. Destacan los estudiantes que el profesor de matemática es exigente $(59,1 \%)$. Se podría decir que "exige, pero es comprensivo y paciente". La cualidad que menos se destaca es ser 
aburrido (15,8\%), más estudiantes lo definen como divertido (37,3\%). En consecuencia, se podría concluir que los estudiantes, en general, reflejan una opinión favorable o positiva sobre las características de su profesor; sin embargo, esto no permite descartar que la variable "características del profesor" no esté influyendo en el aprendizaje.

La mayoría de los encuestados considera que su profesor es exigente, dispuesto al diálogo. Una minoría estima que el profesor es aburrido. Por lo tanto, se podría concluir que el profesor es exigente, está dispuesto al diálogo, es comprensivo y no es aburrido.

Figura 1. Características (en porcentaje) que perciben los estudiantes sobre sus profesores de matemática en el aula

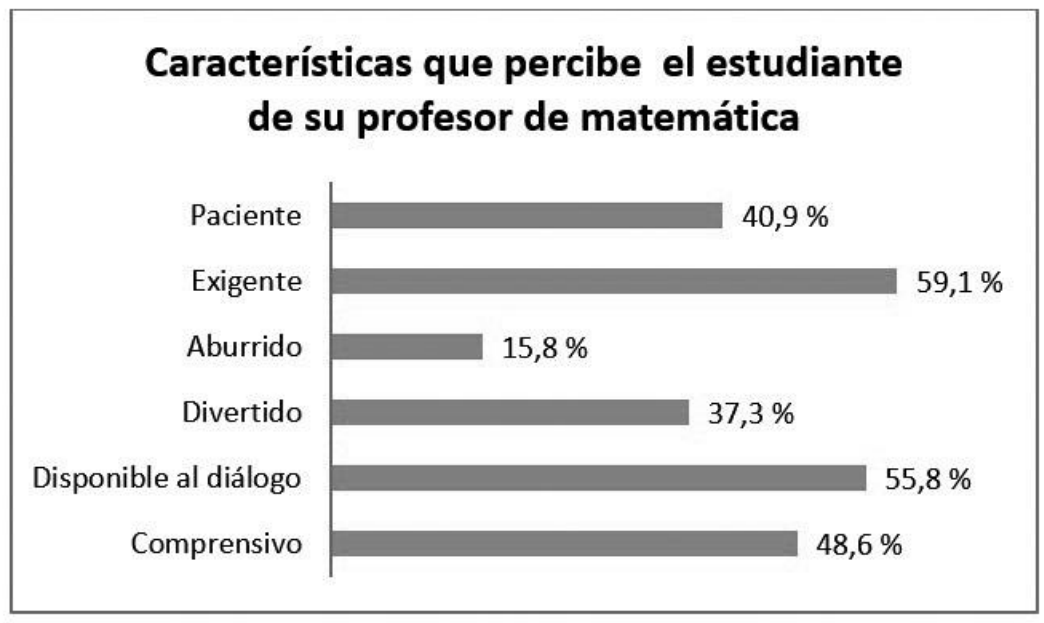

Fuente: Elaboración propia (2016)

\section{Proceso enseñanza-aprendizaje de la matemática en aula}

En la Figura 2 se observa que los estudiantes, en su mayoría, dicen aprender de su profesor (58,3\%). Es menor el porcentaje de estudiantes que afirma aprender de sus compañeros $(22,7 \%)$ o de un grupo de trabajo (18,9\%). Si bien hay coincidencia en que fundamentalmente aprenden del profesor, resulta interesante constatar que algunos afirman que también pueden aprender de sus compañeros. Los resultados reflejan que la figura del profesor es el factor primordial para favorecer el proceso de aprendizaje. 
Figura 2. Porcentaje sobre las fuentes de aprendizaje de la matemática percibidas por los estudiantes

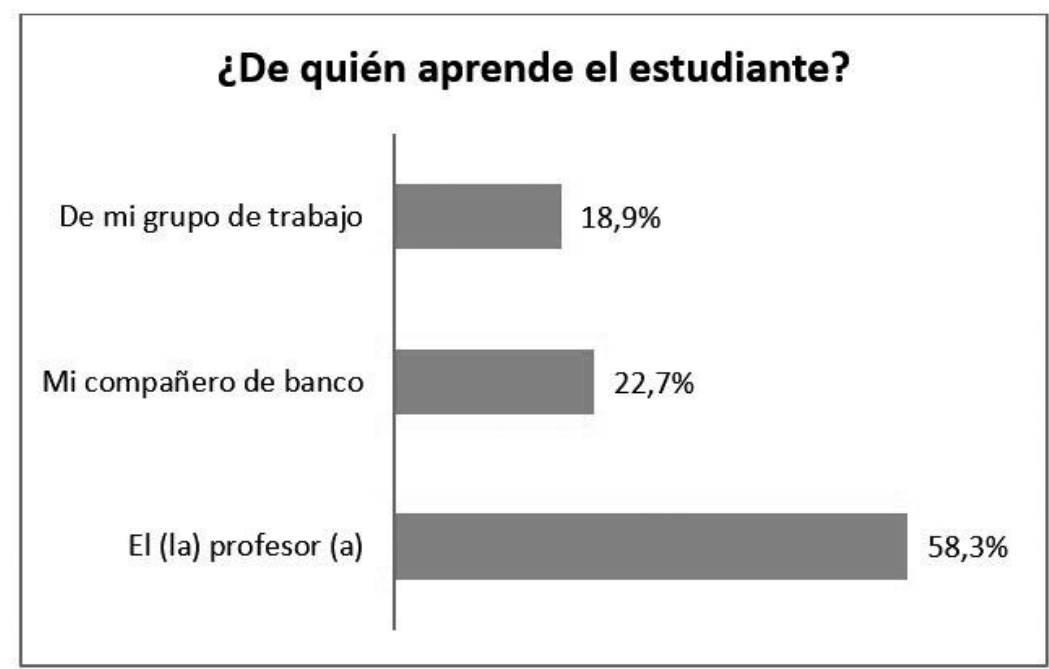

Fuente: Elaboración propia (2016)

De acuerdo con la Figura 3, se observa que los estudiantes, en general, afirman que el profesor se preocupa de todos sus alumnos, les cueste o no les cueste aprender matemática $(70,2 \%)$. Sin embargo, un porcentaje no menor $(20,1 \%)$ observa que sus profesores se preocupan de aquellos a los que les cuesta menos aprender matemática. En cambio, el 9,1\% de los estudiantes expresa que sus docentes se preocupan de aquellos alumnos a los que más les cuesta.

Figura 3. Percepción (en porcentaje) sobre a qué tipo de alumnos dirigen su preocupación los docentes

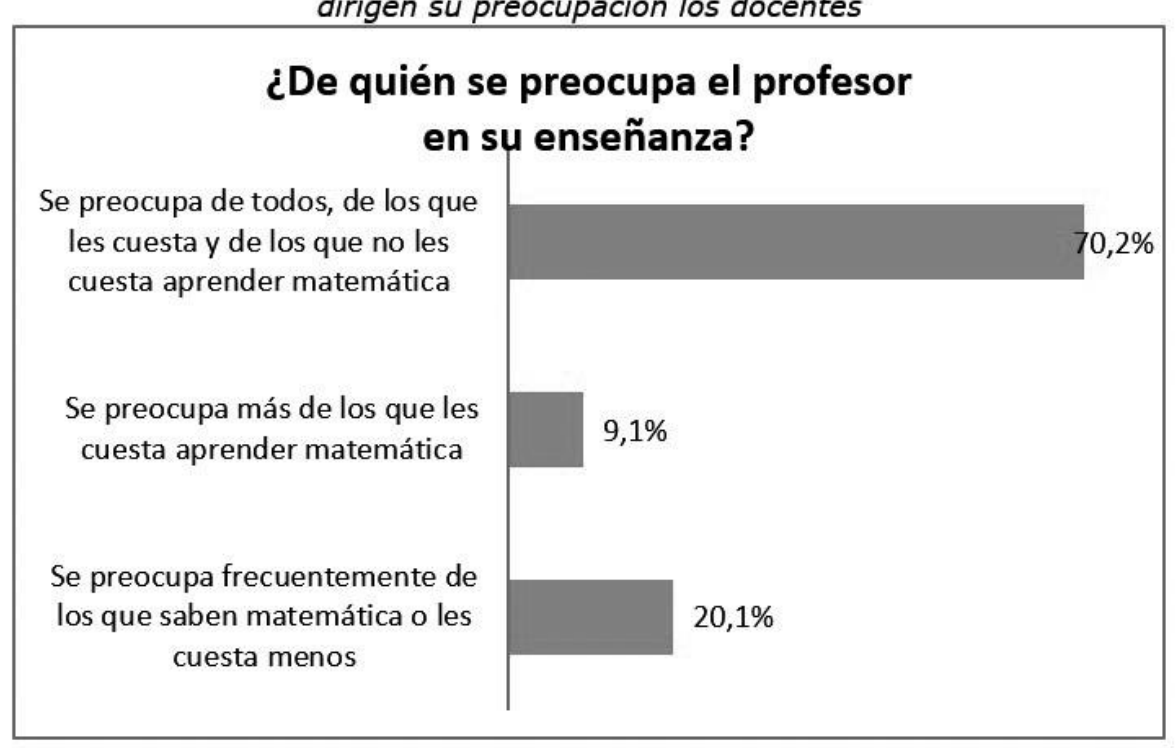

Fuente: Elaboración propia (2016) 
Respecto a las actividades que se plantean en la clase (Figura 4), se advierte que más de la mitad de los estudiantes señala que los ejercicios son fáciles de entender $(57,5 \%)$ y solo un $25,4 \%$ afirma que los encuentra difíciles de comprender. Cuando se les consulta sobre si esos ejercicios (problemas) están relacionados con su vida cotidiana, el $32 \%$ responde que sí. Lo anterior da cuenta de que los estudiantes presentarían niveles muy distintos de desarrollo de la competencia matemática, pues el $57,5 \%$ encuentra que los ejercicios son fáciles. Se asume, por lo tanto, que para estos estudiantes la matemática no implica un grado importante de dificultad y los contenidos les resultan accesibles.

En cualquier caso, un alumno puede tener la impresión de que los ejercicios son fáciles, pero eso no necesariamente se traduce en un buen rendimiento, ya que puede haber otros factores asociados. Cabría esperar, sin embargo, que aquellos que presentan dificultades de aprendizaje de la matemática —o, dicho de otra manera, un menor nivel de competencia matemática - sean quienes opinaron que los ejercicios son difíciles de comprender.

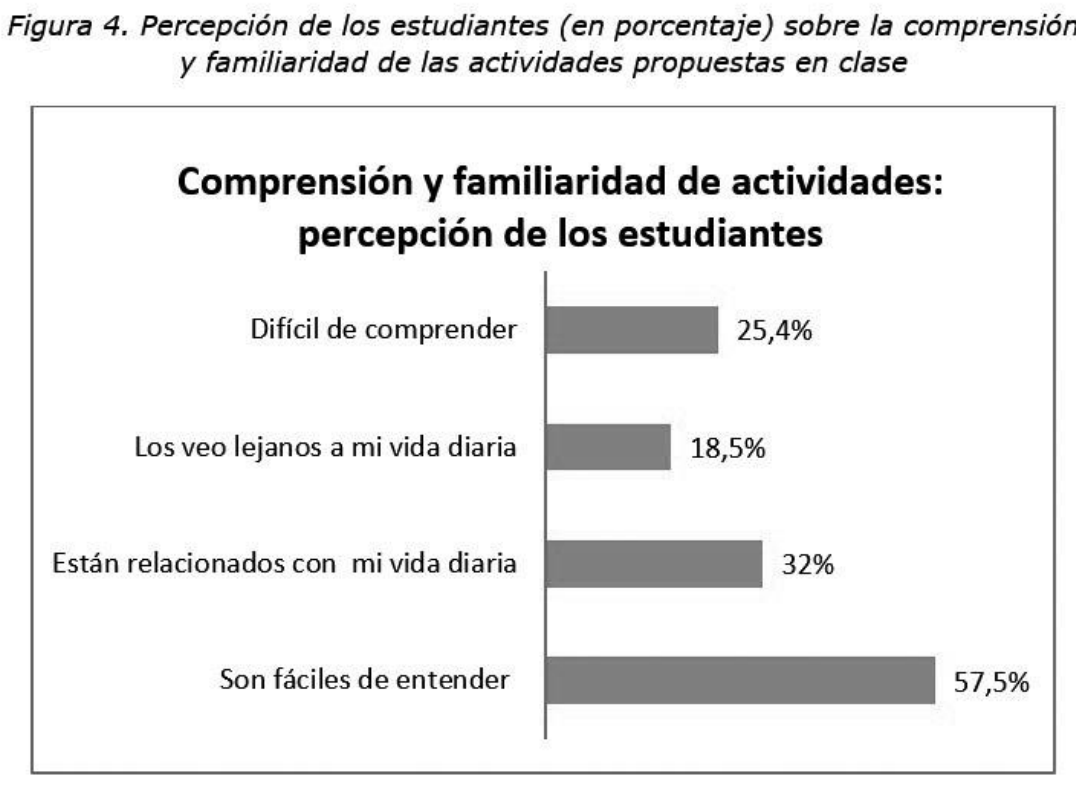

Fuente: Elaboración propia (2016)

Tal como se puede observar en la Figura 5, más de la mitad de los estudiantes $(58,1 \%)$ opina que el profesor siempre o muchas veces propone materias atrayentes, interesantes, importantes. Sin embargo, el $42 \%$ opina que eso ocurre pocas veces o nunca. Si bien las respuestas son favorables en más del $50 \%$ de los casos, esto no resulta así para un porcentaje importante de los estudiantes, situación que podría estar incidiendo en su nivel de aprendizaje. 
Figura 5. Porcentajes de los niveles de interés por las materias enseñadas por los profesores: percepción de los estudiantes

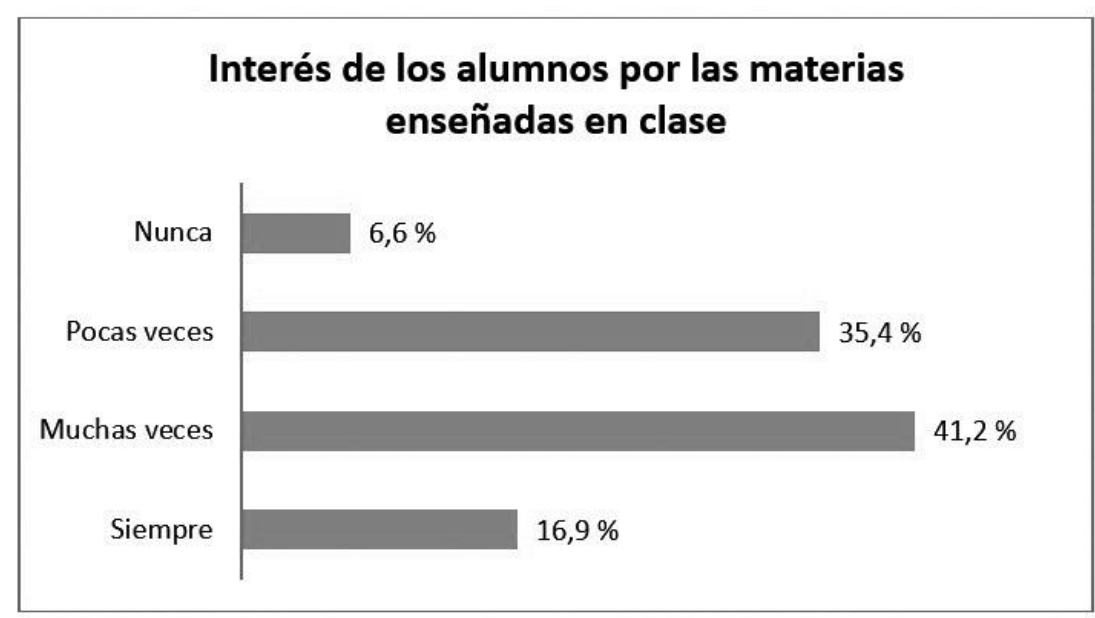

Fuente: Elaboración propia (2016)

Respecto a la interacción del profesor con los estudiantes (Figura 6), estos indican que el docente interactúa bastante o mucho con ellos (24,9\% y $61,9 \%$, respectivamente). Un porcentaje menor señala que lo hace poco (11,6\%). Las respuestas de los estudiantes refieren a un profesor muy sensible al logro de aprendizaje por parte de sus alumnos. Esto sucede en razón de que el profesor, aparentemente, se esforzaría porque sus alumnos aprendan el contenido enseñado, lo que se refleja en que la frecuencia de las interacciones con el grupo, en general, son positivas.

Figura 6. Porcentajes del nivel de interacción del profesor con los estudiantes cuando estos no comprenden las materias

\section{¿Cuánto interactúa el profesor, con los estudiantes, cuando estos no comprenden?}

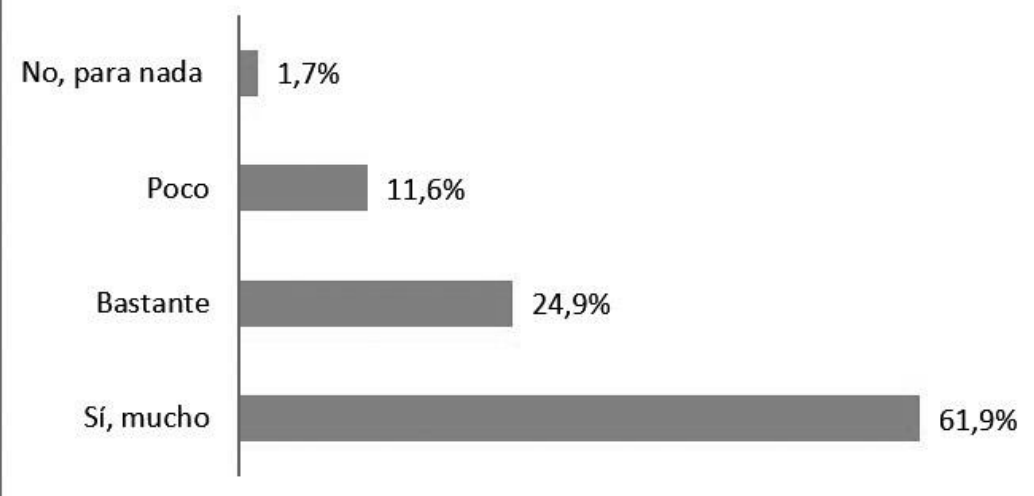

Fuente: Elaboración propia (2016) 
Se observa una tendencia a valorar la capacidad del profesor para explicar un tema de manera distinta (Figura 7), pues el $80,1 \%$ de los alumnos manifiestan que esa capacidad está muy presente. Si se analizan las respuestas de los estudiantes a esta pregunta, queda en evidencia que el profesor no solo repite las explicaciones cuando los alumnos no han entendido algo, sino que, además, diversifica esas explicaciones, aspecto que resulta evidente para la mayoría de los estudiantes.

La diversificación de las explicaciones, o de las verbalizaciones o ayudas que proporciona el profesor, favorece también la comprensión de lo enseñado y la adquisición de conocimientos. En contrapartida, cabe suponer que si esas explicaciones son siempre las mismas y no se diversifican, se están dando pocas oportunidades de aprender a una porción de los alumnos, en especial a aquellos para quienes la materia resulta más compleja o que presentan dificultades para aprender.

Figura 7. Porcentajes sobre la variedad de las explicaciones del docente cuando el estudiante no comprende

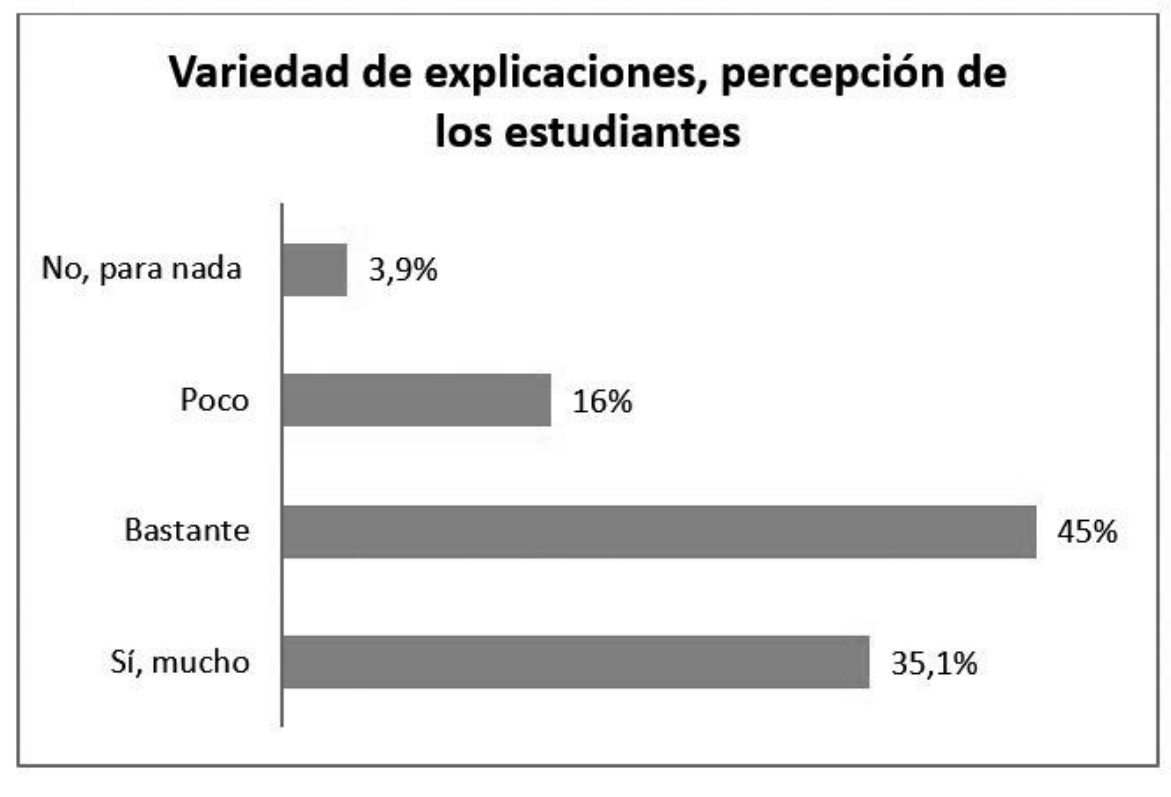

Fuente: Elaboración propia (2016)

Los resultados asociados al interés que genera la forma en que el profesor enseña (Figura 8) muestran que los estudiantes opinan que lo hace de manera llamativa, interesante $(74,6 \%)$. Un $25,4 \%$ expresa una opinión negativa, esto es, que la forma de enseñar del profesor es poco o nada atrayente. 
Figura 8. Porcentajes del nivel de interés que provoca, en los estudiantes, la forma en que los contenidos son enseñados

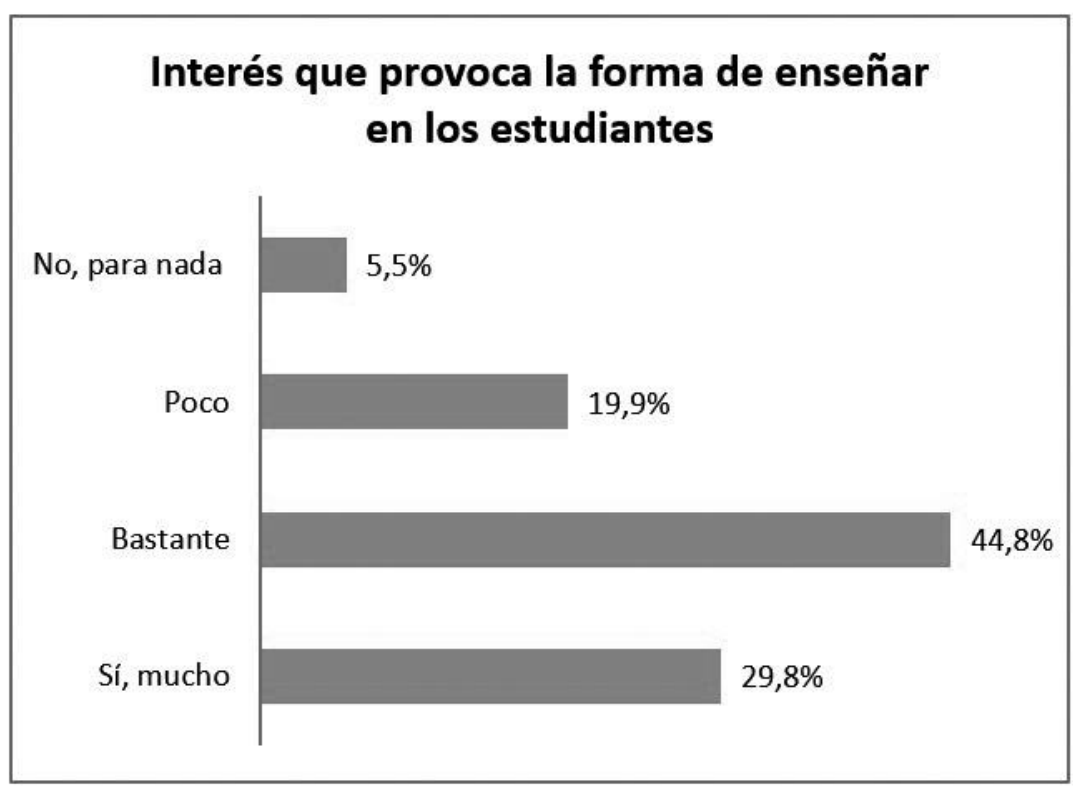

Fuente: Elaboración propia (2016)

La Figura 9 muestra que un $80,7 \%$ de los estudiantes afirma que el profesor lo ha ayudado a aprender, mientras que un 19,3\% señala que eso ha ocurrido pocas veces o nunca. Este resultado coincide con aquel en que se pregunta sobre si se aprende del profesor o de los compañeros, puesto que se observan porcentajes similares de respuesta entre quienes afirman que aprenden fundamentalmente del profesor y quienes señalan que el profesor ha sido quien les ha ayudado a aprender.

Se advierte que se mantiene relativamente constante el porcentaje de alumnos que tiene una percepción negativa de su profesor. Es de esperar que estas opiniones se relacionen con el rendimiento de los estudiantes o con el nivel de dificultad que el aprendizaje de la matemática les reporta; todo esto asociado además con el nivel de interés que experimentan y con su actitud y disposición a aprender. 
Figura 9. Porcentajes sobre la percepción del interés del profesor por que los estudiantes aprendan

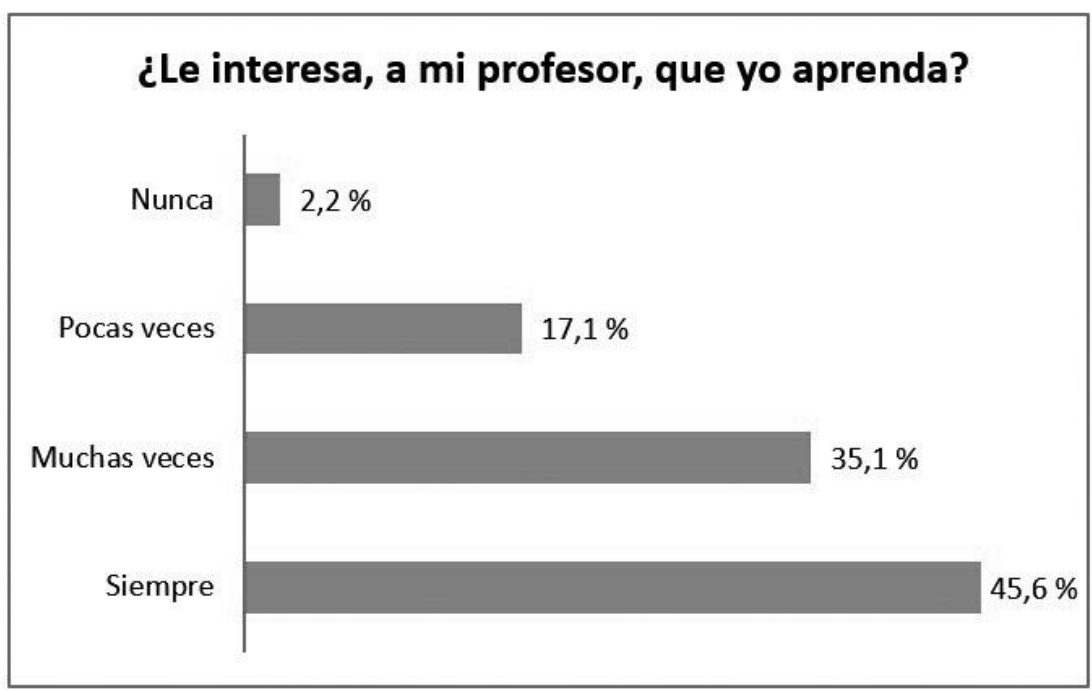

Fuente: Elaboración propia (2016)

En la Figura 10 se describen los resultados asociados al aprendizaje de la matemática. Las respuestas reflejan que es mayor el porcentaje de alumnos que encuentran la asignatura difícil o muy difícil (56,7\%) que los que la encuentran fácil o muy fácil $(43,4 \%)$.

Los resultados ratifican lo que se documenta en otras experiencias relacionadas: son muchos, tal vez demasiados — casi un $60 \%$ en este caso-, quienes ven en la matemática un obstáculo importante y difícil de sobrepasar. Este sentimiento lleva ineludiblemente aparejados las expectativas de los estudiantes y su sensación de autoeficacia, y se extrema entre quienes tienen dificultades en el aprendizaje de la matemática o que han sufrido experiencias de fracaso y frustración en esta área de conocimiento (Corica y Otero, 2007).

De cualquier forma, a la luz de los resultados, parecería que incluso tienen una opinión similar aquellos alumnos que poseen un regular o buen rendimiento en la asignatura, o al menos una porción de ellos. 
Figura 10. Porcentajes del nivel de complejidad del aprendizaje de la matemática, según los estudiantes

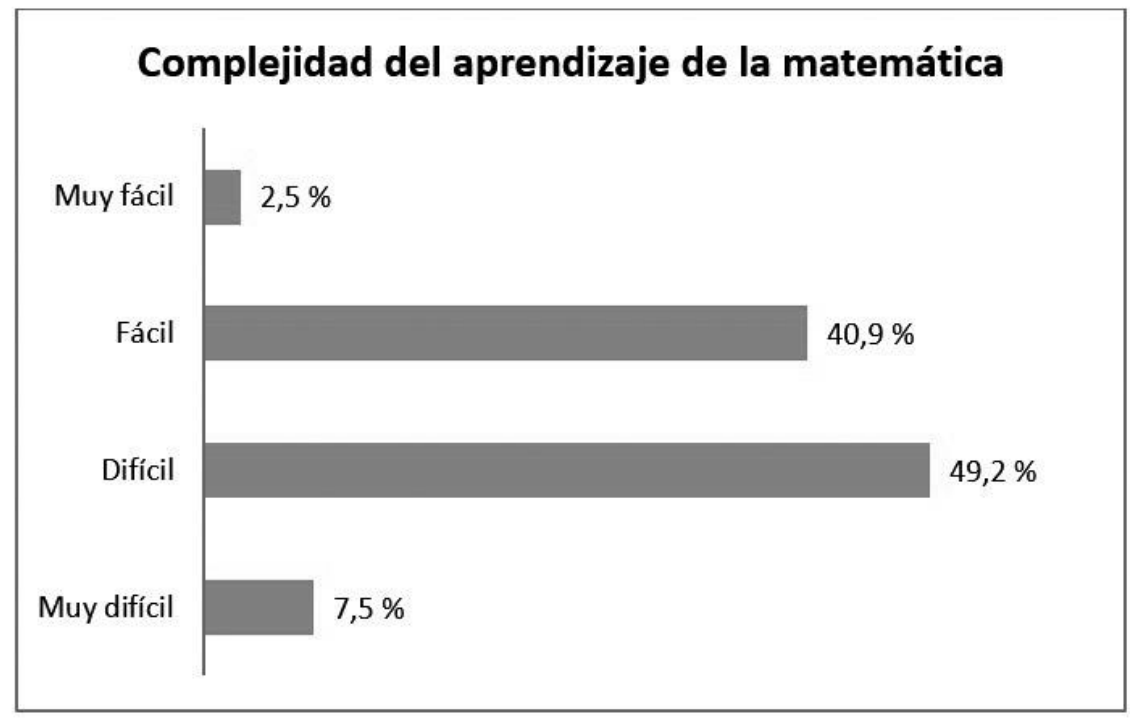

Fuente: Elaboración propia (2016)

\section{DISCUSIÓN Y CONCLUSIONES}

El estudio da cuenta de la percepción de los estudiantes de fin de segundo ciclo de Enseñanza Básica sobre algunas características del profesor de matemática y el proceso de enseñanza-aprendizaje de esta asignatura en el aula.

Tal como se ha planteado en el marco referencial de este trabajo, entre los factores que determinan la calidad de los aprendizajes se encuentran las estrategias de enseñanza que el profesor utiliza. En consecuencia, lo que se requiere para que los alumnos alcancen mayores niveles de competencia matemática es que los profesores modifiquen sus estrategias didácticas. Se asume que unas adecuadas estrategias de enseñanza favorecen el desarrollo de una determinada competencia y que, por el contrario, la utilización de estrategias inadecuadas dificulta el aprendizaje, impide u obstaculiza el desarrollo de una determinada competencia o simplemente genera fracaso. En síntesis, las características del profesor y, en especial, los modelos didácticos - las estrategias de enseñanza - posibilitan o dificultan el aprendizaje.

A modo de conclusión, se resumen los hallazgos centrales recogidos a partir de la opinión de los estudiantes sobre los aspectos asumidos en esta perspectiva de análisis. Estos hallazgos van en la línea con lo que exponen diversos autores acerca de la importancia que tienen las estrategias didácticas y otras características del profesor para el logro de los aprendizajes. 
En relación a cómo caracterizan los estudiantes a sus profesores de matemática, se concluye que estos les asocian características que podrían definirse como idóneas o deseables, y que tienen una valoración más bien positiva de sus docentes. Por ejemplo, señalan que en general son exigentes, que se muestran dispuestos al diálogo, que son comprensivos y no son aburridos. Del mismo modo, expresan que están satisfechos con lo que sabe el profesor sobre los temas de matemática y el dominio que tiene de la asignatura.

Los estudiantes refieren además a un profesor muy sensible al logro de aprendizaje, que aparentemente se esfuerza por que sus alumnos aprendan el contenido enseñado. La frecuencia de las interacciones del docente con el grupo es vistas como positiva; esto refleja que el profesor tiene como propósito la comprensión de lo enseñado, asumiendo que estas interacciones constituyan verdaderas ayudas para la adquisición de conocimientos.

Queda en evidencia también que el profesor no solo repite las explicaciones cuando los alumnos no han entendido algo, sino que además diversifica esas explicaciones, aspecto que identifica la mayoría de los estudiantes. Esta diversificación, como se mencionó, favorece la comprensión de lo enseñado y la adquisición de conocimientos. Asimismo, los estudiantes constatan que el profesor destina atención y preocupación a todos los estudiantes de su clase, les cueste o no les cueste aprender matemática.

Otra conclusión relevante del estudio tiene que ver con la percepción del nivel de dificultad del aprendizaje de la materia. Los datos coinciden con lo que otros autores han planteado: casi un $60 \%$ de los encuestados para este trabajo consideran que la matemática es una asignatura difícil. Por otra parte, el porcentaje de estudiantes que encuentra fáciles o muy fáciles los ejercicios de clase es muy similar al porcentaje anterior; solo un $25 \%$ encuentra que estos ejercicios son difíciles de comprender.

Se podría suponer que, si la materia es percibida como difícil, también deberían serlo los ejercicios o actividades que son enseñados. Sin embargo, esta relación no se observa en este caso. Resulta difícil explicar esta contradicción; solo puede aventurarse una razón, vinculada con el hecho de que podría haber factores culturales mediando las respuestas de los alumnos. Por la edad que tienen, estos ya han estado expuestos a comentarios u opiniones de diversas fuentes, que pueden dar cuenta de las malas experiencias de muchas personas con la matemática, de lo difícil que resulta para muchos estudiantes y de los deficientes resultados que arrojan distintas evaluaciones estandarizadas en el ámbito nacional. Esto puede chocar con sus propias vivencias y llevarlos a afirmar que, a pesar de la dificultad de la asignatura, las tareas presentadas en clase son más bien fáciles.

Finalmente, es necesario aclarar que si bien la muestra que se consideró en la investigación es importante (casi 800 estudiantes), no es aconsejable generalizar los resultados a otras poblaciones. 


\section{REFERENCIAS}

Badano, C., y Dodera, M. (1999). Un estudio de la influencia de la representación de la matemática en el rendimiento académico del alumno de primer año de la Universidad. Educación Matemática, 11(3), 79-88.

Blanco, L. (1997). Tipos de tareas para desarrollar el conocimiento didáctico del contenido. En L. Rico y M. Sierra (Eds.), Primer Simposio de Sociedad Española de Investigación en Educación Matemática (pp. 34-40). Zamora, España: Sociedad Española de Investigación en Educación Matemática.

Bolea, P., Bosch, M., y Gascón, J. (2001). La transposición didáctica de organizaciones matemáticas en proceso de algebrización: el caso de la proporcionalidad. Recherches en Didactique des Mathématiques, 23(1), 79-136.

Corica, A. (2007). El saber matemático, su enseñanza y su aprendizaje: la mirada de alumnos y profesores (Tesis de Grado). Universidad Nacional del Centro de la Provincia de Buenos Aires, Buenos Aires, Argentina.

Corica, A., y Otero, M. (2007). Las ideas de algunos estudiantes acerca de la enseñanza-aprendizaje de la Matemática en el Nivel Medio. Revista Electrónica de Investigación en Educación en Ciencias, 2(1), 40-68.

Gascón, J., Bosch, M., y Bolea, P. (2001). ¿Cómo se construyen los problemas en didáctica de las matemáticas?. Educación Matemática, 13(3), 22-63.

Hernández, R., Fernández, C., y Baptista, P. (2010). Metodología de la Investigación. México, D. F., México: McGraw Hill.

Hidalgo, S., Maroto, A., y Palacios, A. (2004). ¿Por qué se rechazan las Matemáticas? Análisis evolutivo y multivariante de actitudes relevantes hacia las Matemáticas. Revista de Educación, 334, 75-95.

Labarca, A. (2001). Métodos de Investigación en Educación. Santiago de Chile, Chile: Universidad Metropolitana de Ciencias de la Educación.

Míguez, M. (2004). El rechazo hacia las matemáticas. Una primera aproximación. En L. Díaz (Ed.), Acta Latinoamericana de Matemática Educativa 17 (pp. 292-298). México, D. F., México: Comité Latinoamericano de Matemática Educativa A. C.

Organización de Estados Iberoamericanos. (2015). Cosas de la educación. San José, Costa Rica: Universia.

Organización para el Crecimiento y el Desarrollo Económico. (2004). Learning for Tomorrow's World: First Results from PISA 2003. Paris, Francia: Organización para el Crecimiento y el Desarrollo Económico.

Otero, M. R., Fanaro, M., y Elichiribehety, I. (2001). El conocimiento matemático de los estudiantes que ingresan a la Universidad. Revista Latinoamericana de Investigación en Matemática Educativa, 4(3), 267-287. 
Prado, M., Navarro, Berguido, S., y De la Cruz, J. (2013). El por qué de la apatía a la matemática (Tesis de Maestría). Universidad de Panamá, Panamá.

Reimers, F. (2006). Aprender más y mejor. Políticas, programas y oportunidades de aprendizaje en Educación Básica en México. México, D. F., México: Secretaría de Educación Pública - Fondo de Cultura Económica.

Rojas, G., y Cortés, J. (2002). La calidad académica vista por los estudiantes. Revista de la Educación Superior, 122, 49-63.

Sepúlveda, A., Opazo, M., Díaz-Levicoy, D., Jara, D., Sáez, D., y Guerrero, D. (2016). ¿A qué atribuyen los estudiantes de Educación Básica la dificultad de aprender matemática?. Revista de Orientación Educacional, 31(58), 105-119.

Sepúlveda, A., Payahuala, H., Lemarie, F., y Opazo, M. (2017). ¿Cómo evalúan el aprendizaje los profesores de matemática?: percepción de los estudiantes de escuelas básicas municipalizadas de la décima región. Revista de Estudios y Experiencias en Educación, 16(30), 63-79.

Tejedor, F. J. (2003). Un modelo de evaluación del profesorado universitario. Revista de Investigación Educativa, 21(1), 157-182. 